\title{
Correlation between full-field measurements and numerical simulation results for multiple delamination composite specimens in bending
}

\author{
C. Devivier ${ }^{1 \& 2, a}$, D. Thompson ${ }^{2, b}$, F. Pierron ${ }^{1, c}$ and M.R. Wisnom ${ }^{2, d}$ \\ ${ }^{1}$ Laboratoire de Mécanique et Procédés de Fabrication, Arts et Métiers ParisTech, Centre de \\ Châlons-en-Champagne, Rue St Dominique, BP 508, 51006 Châlons-en-Champagne Cedex, \\ France \\ ${ }^{2}$ Advanced Composites Centre for Innovation and Science, Queen's Building, University of Bristol, \\ University Walk, Bristol, BS8 1TR, United Kingdom \\ adevivier@ensam.fr, 'baniel.thompson@bristol.ac.uk, ${ }^{c}$ fabrice.pierron@chalons.ensam.fr, \\ dm.wisnom@bristol.ac.uk
}

Keywords: Damage, full-field, strains, deflectometry, composite

\begin{abstract}
This paper studies the effect of delaminations on strain maps for a simple cantilever beam. The aim is to build an experimental set-up which allows detecting very slight modifications in the strain maps. The case studied is a single delamination on the mid-plane. The measurement method is the deflectometry technique which enables direct slope measurements on a reflective specimen. The comparison with finite element models clearly indicated that the surface strains bear the information of the extent of the delamination. The second step is to use these surface strains to identify a stiffness reduction map for real impact damages.
\end{abstract}

\section{Introduction}

In aeronautical engineering, many surfaces can be assimilated to beam- or plate-like structures like the wings or the blades. In these structures, damage like delamination affects strongly the global behaviour and especially its stiffness hence its remaining life. Therefore, detecting this damage is a crucial point for safety reasons. A large amount of work has been done on this subject. Among the studied techniques, one can find natural frequency changes, mode shapes (changes or derivatives) and modal damping. However, as damage can be slight, the modification of these indicators may not be enough [1]. A summary of the vibration property features applied to damage identification algorithms was given in [2].

As the modifications in the fields caused by the inserted defects are very slight, monitoring of the variation of strains is preferred to that of displacements, so techniques such as curvature mode shape, modal or spectral strain energy and strain frequency response function can be used. All of them use vibrations, but it is also possible to use static techniques such as interferometric methods, digital image correlation (DIC) or deflectometry. Interferometry has a very good resolution but it is very sensitive to any disturbances such as vibrations [3]. DIC requires two differentiations to obtain the strains, therefore the noise will be strongly emphasized. The deflectometry technique requires only one differentiation and is insensitive to vibrations. This paper describes a correlation between numerical and experimental strain fields in bending for a cantilever beam. In the first part the set-up of the measurement post-processing is explained, then the numerical model is described and finally the results are presented and discussed.

\section{Experimental set-up and strain extractions}

The deflectometry is based on the specular light reflection principle and its set-up is shown in Fig.1. The specimen is a cantilever beam loaded with $5.5 \mathrm{~N}$ on the free end as shown in Fig. 2. The 
distance $h$ is $1.23 \mathrm{~m}$, the grid pitch of the reflected grid is $1 \mathrm{~mm}$ and the samples are $250 \mathrm{~mm}$ long, $50 \mathrm{~mm}$ wide and $4 \mathrm{~mm}$ thick. With these parameters, 8 pixels described exactly one period of the grid. The pictures were taken by a 12 Mpixels Nikon D90 camera with a Nikkor $105 \mathrm{~mm}$ lens. A noise analysis done with this set-up gave a resolution of $2.510^{-6} \mathrm{rad}$. The resolution is the standard deviation of the difference between two phase maps at rest, multiplied by the sensitivity. Also because of the clamp, there is no measurement for a $2 \mathrm{~mm}$-width band across the whole width.

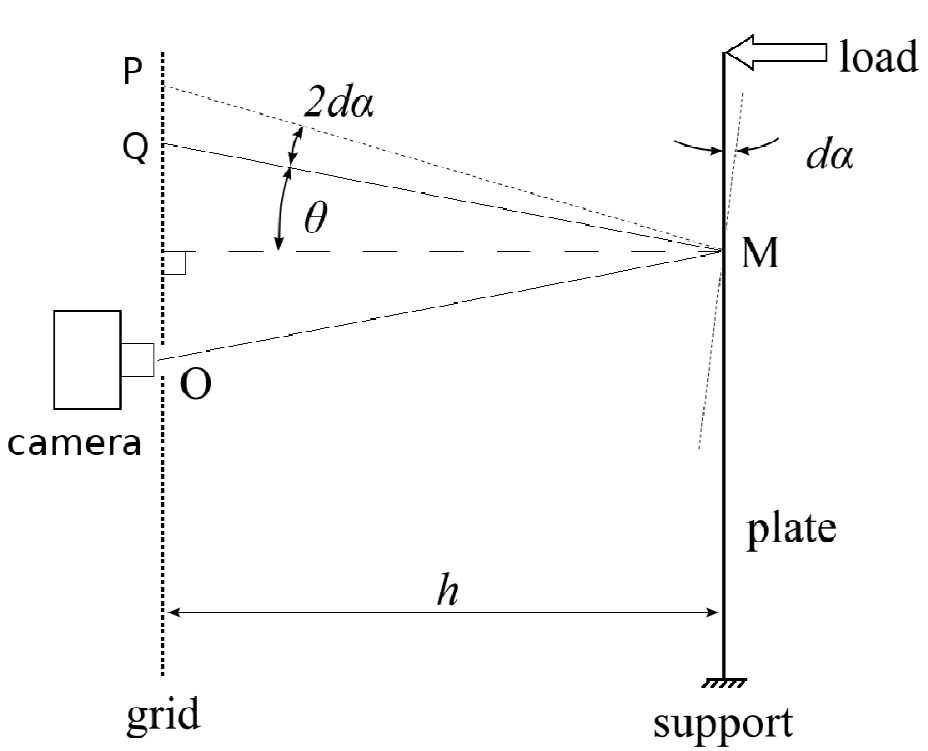

Figure 1: Principle of deflectometry

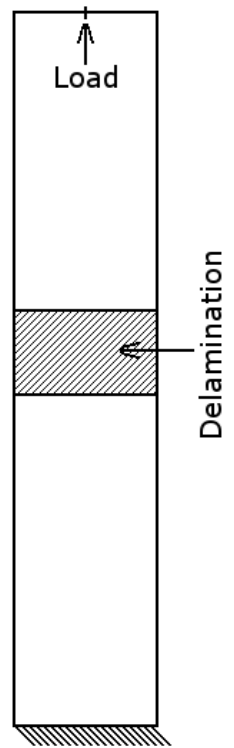

Figure 2: Model

The method consists of observing the reflection of a grid on the surface of the specimen. As composite components do not have enough specular reflection, a resin (Sicomin Surf Clear) is applied to make a thin reflective coating. At rest, the CCD sensor receives the light coming from $\mathrm{P}$ after reflection on $\mathrm{M}$. Once the specimen has been loaded, the slope at the point $\mathrm{M}$ has been modified by $\mathrm{d} \alpha$ so the sensor records the light coming from $\mathrm{Q}$. Therefore, the camera observes a deformation of the grid. This deformation will be evaluated using spatial phase stepping at rest and under loading for each direction. The phase at rest will be subtracted from the phase maps in the loaded state to obtain the phase modification introduced only by the loading. As these two phase maps are wrapped, an unwrapping process is needed. For that part, a routine developed by BioucasDias and Valadao [4] has been used.

An example of a fringe analysis is shown in Fig. 3. Fig. 3 (a) shows the intensity field of the unloaded beam on the left and the field for the loaded case on the right. From these two states, the phases (transverse and longitudinal) are extracted using a spatial phase stepping algorithm, Fig.3 (b). For each direction, the phase at rest is subtracted from the phase in the loaded state, Fig. 3 (c). As the obtained phase maps are wrapped, the phases are then unwrapped Fig. 3 (d). The results are divided by the sensitivity to scale them, as in [5]. To obtain the curvatures, the slopes are differentiated using a routine developed by [6] with a radius of 15 pixels. As it fits locally the slopes with a second order polynomial function, it eliminates the high frequency noise from the grid and smoothes the results. The curvatures are multiplied by half the thickness to obtain the strains, based on thin plate theory.

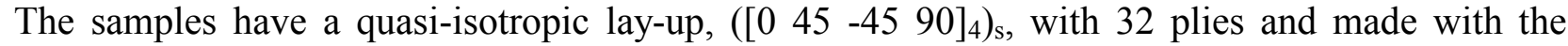
IM7/8552 prepreg. The ply thickness is $0.125 \mathrm{~mm}$. The delaminations inserted are made from a single layer of PTFE film with a thickness of $30 \mu \mathrm{m}$. They are 3 types of samples: undamaged, ones with a $30 \mathrm{~mm}$ delamination across the whole width and with a $50 \mathrm{~mm}$ delamination across the whole 
width. For each type of specimen, 2 samples have been manufactured. The delaminations have been positioned in the centre of the studied area, the striped zone in Fig. 2.

(a)
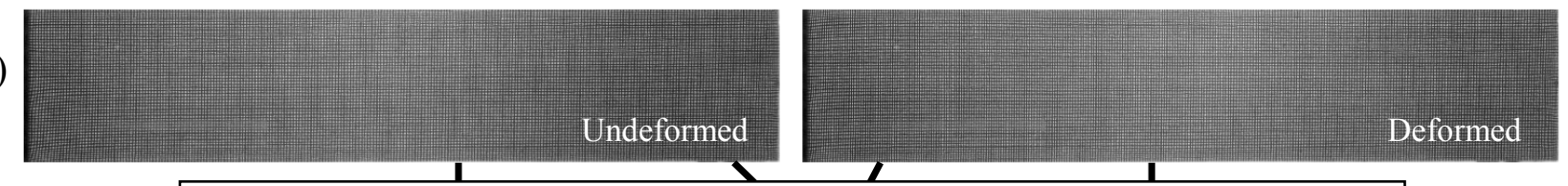

Phase extraction

(b)
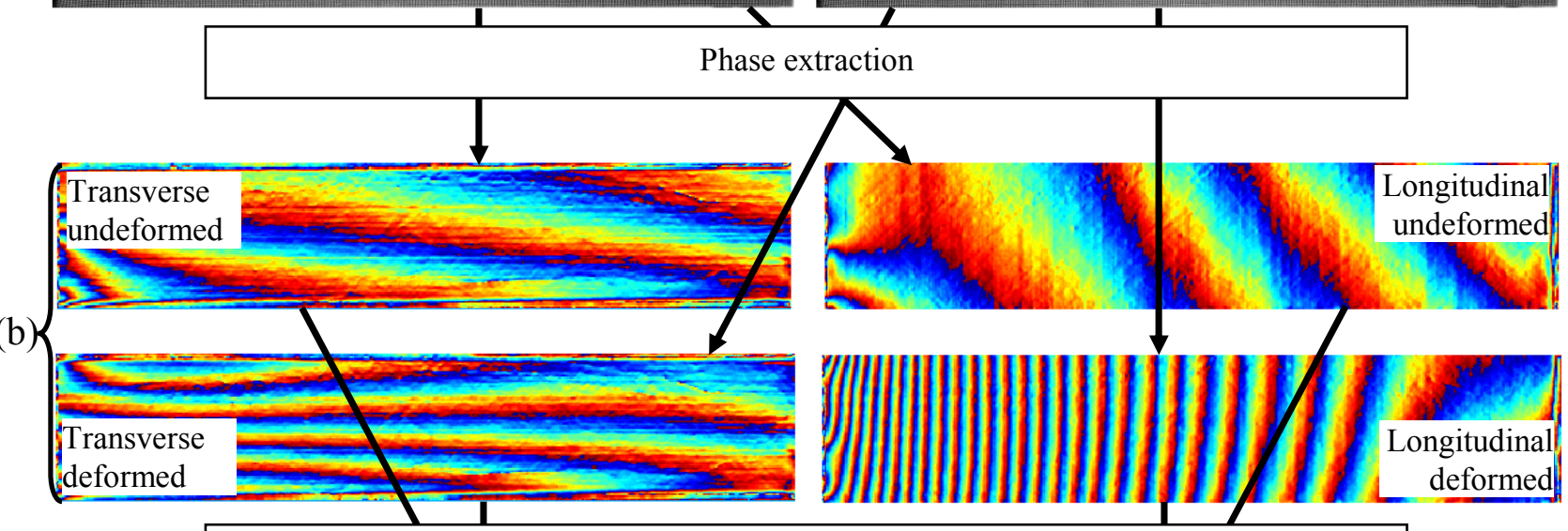

(c)

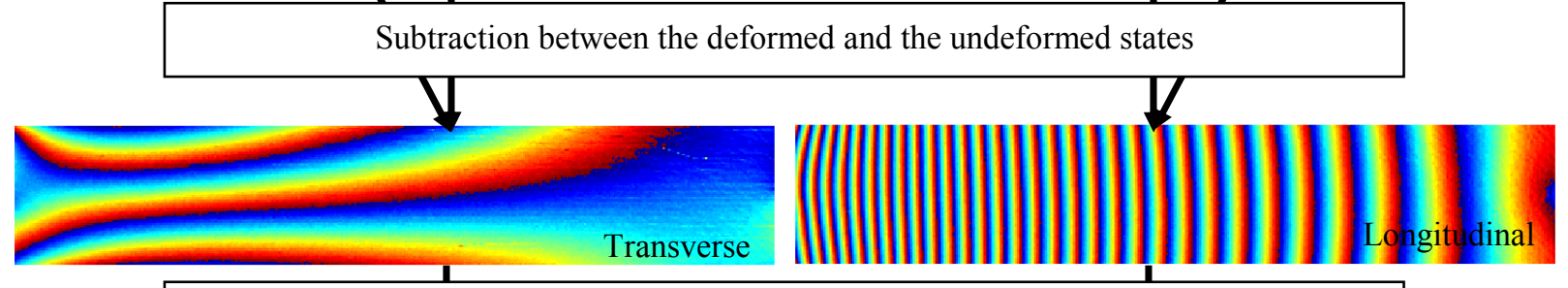

(d)

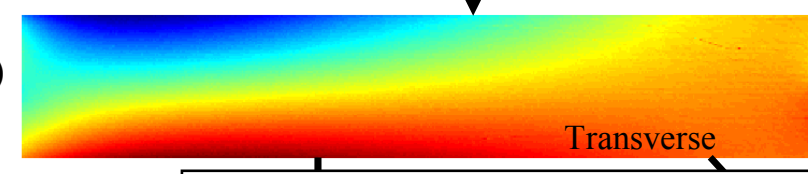

Unwrapping process and scaling
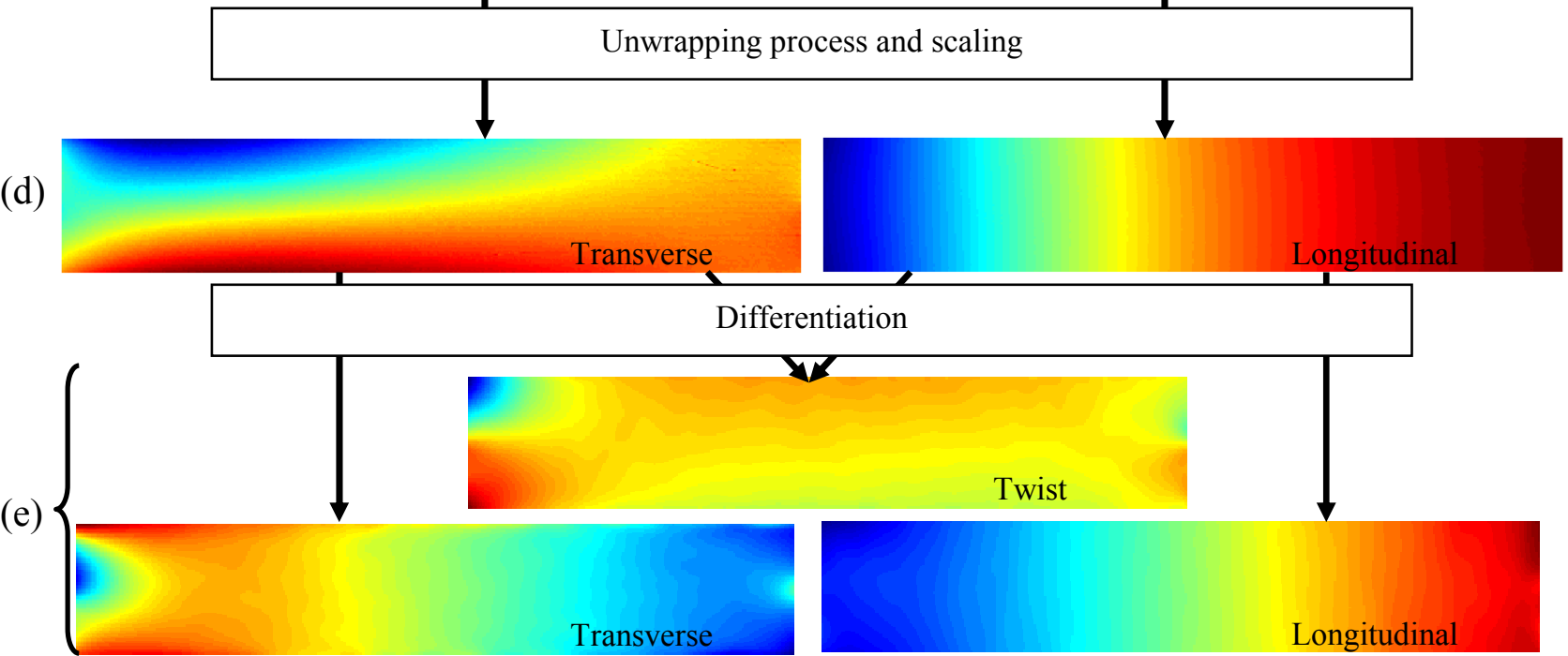

Figure 3: Fringe analysis (a) Intensity pattern (b) phase field (c) corresponding wrapped phase map (d) unwrapped phase maps (e) curvatures maps

\section{Numerical model}

The finite element model uses 8 nodes linear elastic bricks, named C3D8R in Abaqus. The element is $1 \mathrm{~mm}$ long, $1 \mathrm{~mm}$ wide and the thickness depends on the layer as shown in Fig. 4. Each thin element is $0.125 \mathrm{~mm}$ thick, and each thick one is $1.75 \mathrm{~mm}$ which is 14 plies. This way of modelling has been chosen to speed up the computation as the thick elements only transfer the shear stress. The material properties are shown in Table 1. 
The longitudinal and through-the-thickness translations have been blocked at the cantilever end. At the free end, a $5.5 \mathrm{~N}$ load perpendicular to the surface is applied on a single point on the central node. To model the delamination, coincident nodes are unconstrained.

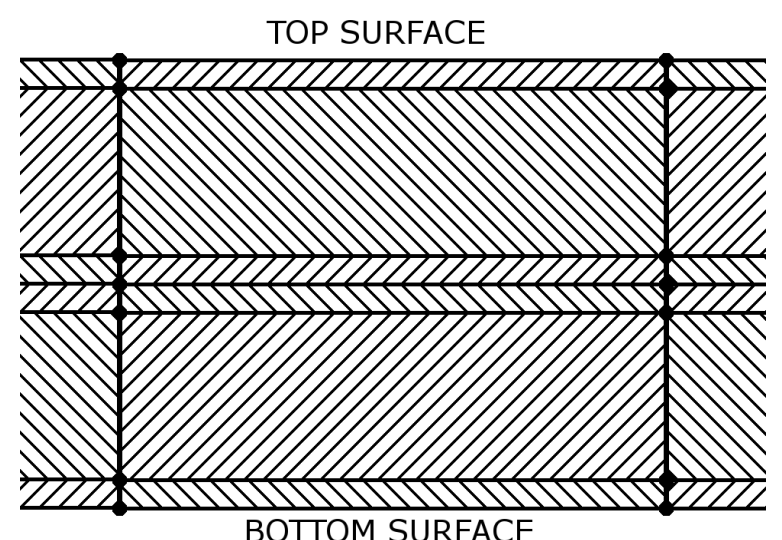

Figure 4: Side view of the meshing. Each striped zone represents an element.

\begin{tabular}{|c|c|}
\hline $\mathrm{E}_{1}$ & $61.6 \mathrm{GPa}$ \\
\hline $\mathrm{E}_{2}$ & $61.6 \mathrm{GPa}$ \\
\hline $\mathrm{E}_{3}$ & $11.3 \mathrm{GPa}$ \\
\hline $\mathrm{G}_{12}$ & $23.4 \mathrm{GPa}$ \\
\hline $\mathrm{G}_{13}$ & $3.92 \mathrm{GPa}$ \\
\hline $\mathrm{G}_{23}$ & $3.92 \mathrm{GPa}$ \\
\hline $\mathrm{v}_{12}$ & 0.319 \\
\hline $\mathrm{v}_{13}$ & 0.319 \\
\hline $\mathrm{v}_{23}$ & 0.319 \\
\hline
\end{tabular}

Table 1: Material properties.

Subscript 1 stands for longitudinal, 2 for transverse and 3 for through the thickness

\section{Single delamination in mid-plane}

The following graphs represent the strain fields in microstrains. Each group of graphs (e.g. Fig. 5.a) has the same colour scale which is indicated only once. The zone where there is no information in the measurements has been deleted in the FE results.

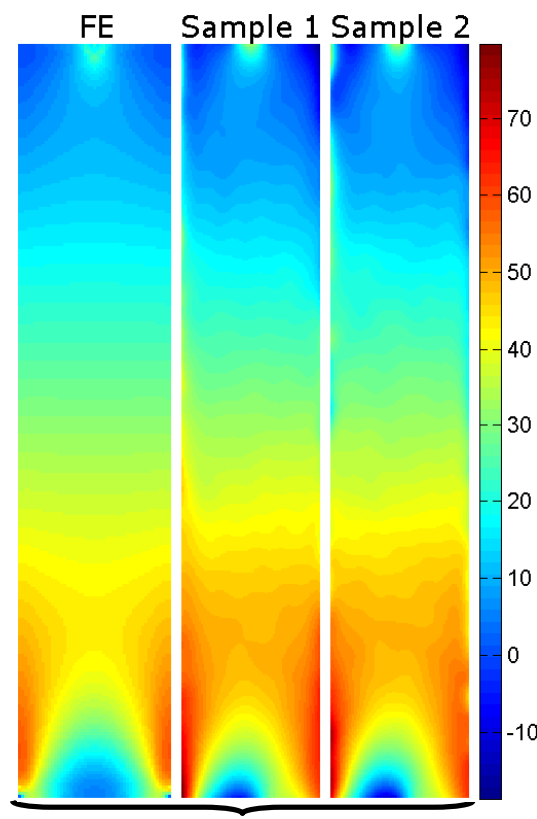

(a)

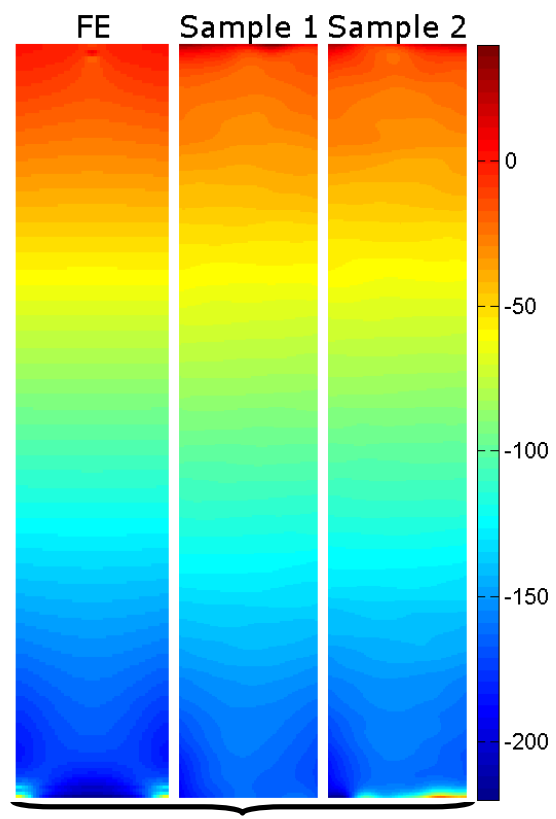

(b)

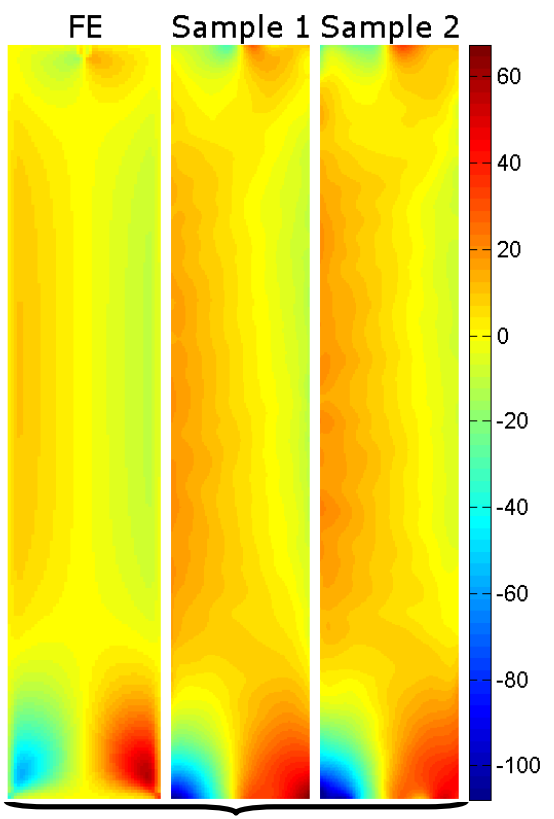

(c)

Figure 5: Strain fields divided by load for an undamaged beam, (a) Transverse strains (b) Longitudinal strains (c) Twist strains

For the undamaged beam, there is very good correlation. The small discrepancy at the root on the longitudinal strains is explained in part by the fact that the applied load was not exactly $5.5 \mathrm{~N}$ and also that the material used did not have exactly the properties used in the FE model. 


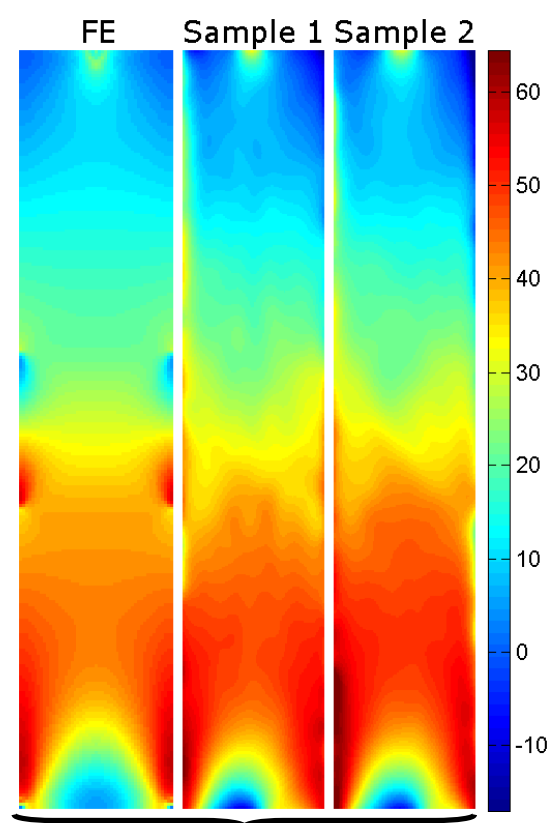

(a)

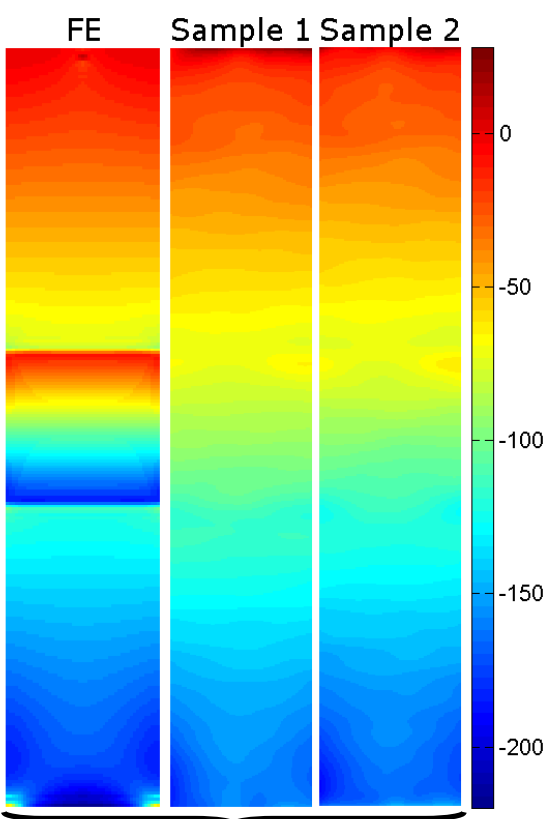

(b)

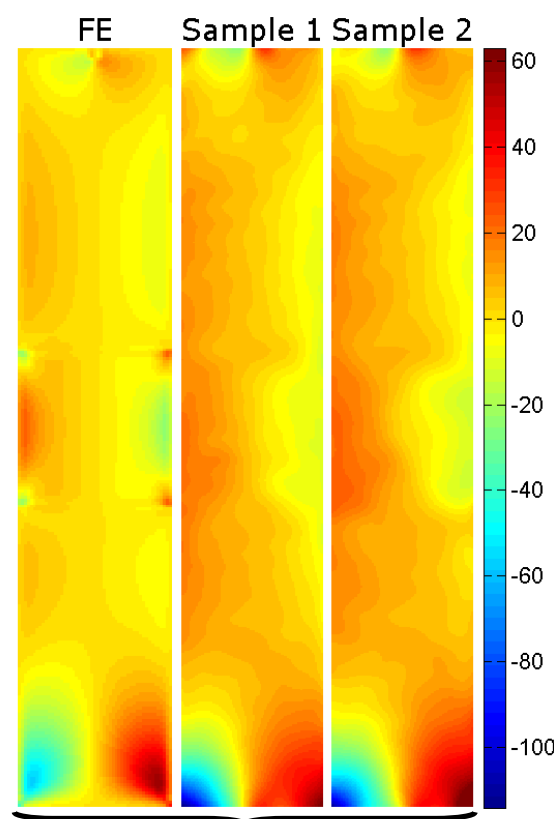

(c)

Figure 6: Strain fields divided by load for a beam with a 50mm delamination, (a) Transverse strains (b) Longitudinal strains (c) Twist strains

From the FE results, the delamination is the most visible in the longitudinal strain maps but the experimental results do not show it. In the twist strains, the pattern predicted by the FE model has been seen. As the delamination has been realised by a single layer of PTFE film, it is possible that the resin stuck to the film.

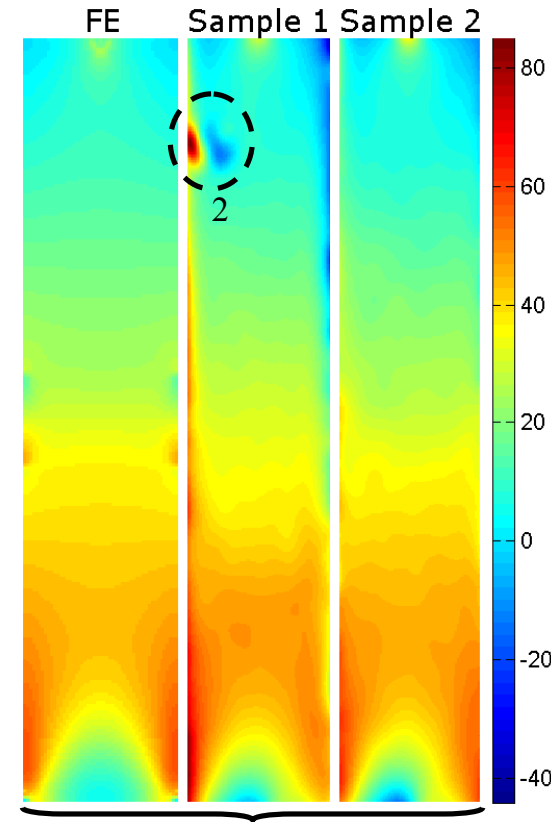

(a)

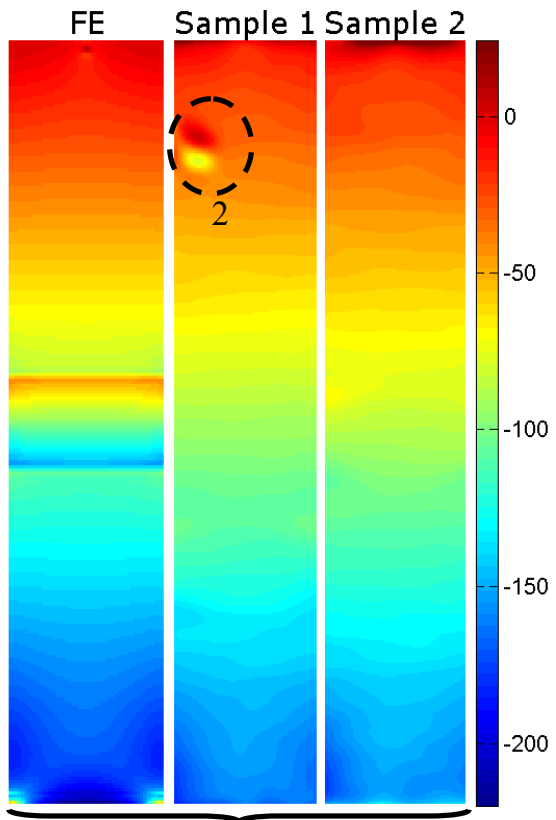

(b)

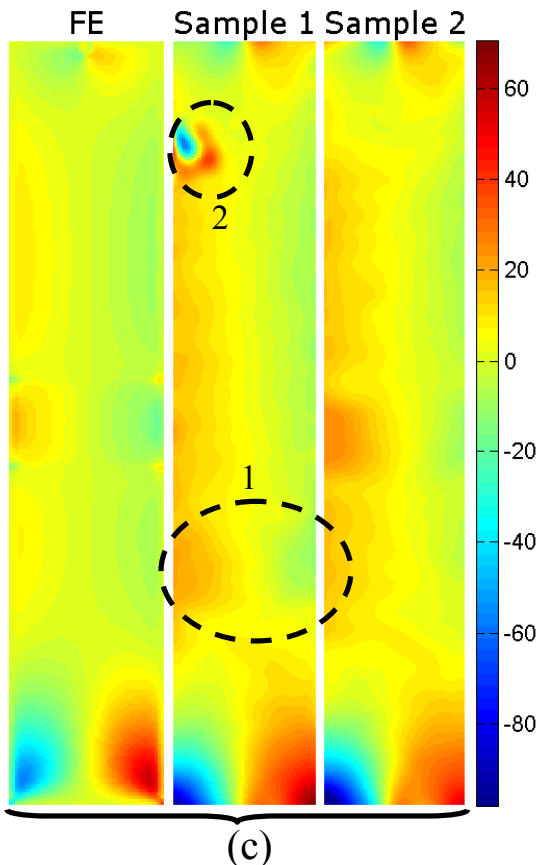

(c)

Figure 7: Strain fields divided by load for a beam with a 30mm delamination, (a) Transverse strains (b) Longitudinal strains (c) Twist strains

For "sample 1" with the 30mm delamination, it appears from the Fig. 7 (c) that the delamination is not centred (circled zone number 1). This was confirmed by examining the specimen again. On the same sample, there was a defect in the coating (circled zones number 2) therefore the missing information was interpolated by the derivative routine. 
These results showed a modification in the strain fields due to the delamination. The modifications are clearly visible for the twist strains and less clear for the longitudinal ones.

\section{Conclusions}

This paper showed a good correlation between the numerical results and the measurements for 2 out of 3 strain components. For these two strain components, a delamination can be clearly identified and located.

This study needs to be broadened to include other cases such as multiple delaminations or real barely visible impact damage. The results from these test measurements can be used with a virtual field routine to extract the orthotropic bending stiffnesses of the damaged and undamaged zones. These strain maps could potentially be used to identify interply properties such as the location, extent and depth of delamination damage. Each of these delamination parameters is potentially uniquely identifiable in the features of the surface strain map.

\section{Acknowledgements}

Daniel Thompson would like to acknowledge Rolls-Royce Plc for their support for his research.

\section{References}

[1] S.W. Doebling, C.R. Farrar, M.B. Prime, A summary review of vibration-based damage identification methods, The Shock and Vibration Digest, vol.30, pp.91-105, 1998

[2] L.S. Lee, V.M. Karbhari, C. Sikorsky, Investigation of integrity and effectiveness of RC bridge deck rehabilitation with CFRP composites, Report No. SSRP-2004/08, Department of Structural Engineering, University of California, San Diego, 2004

[3] Y. Surrel, Deflectometry: a simple and efficient noninterferometric method for slope measurement, Xth SEM International congress on experimental mechanics, 2004

[4] J.M. Bioucas-Dias, G. Valadao, Phase Unwrapping via Graph Cuts, IEEE Transactions on image processing, vol.16, no.3, pp.698-709, March 2007

[5] Kim J.-H., Pierron F., Wisnom M., Syed-Muhamad K., Identification of the local stiffness reduction of a damaged composite plate using the virtual fields method, Composites Part A: Applied Science and Manufacturing, vol. 38, n 9, pp. 2065-2075, 2007.

[6] Avril S., Feissel P., Pierron F., Villon P., Comparison of two approaches for controlling the uncertainty in data differentiation: application to full-field measurements in solid mechanics, Measurement Science and Technology, vol. 21, 015703 (11 pp), 2010. 